\title{
Association of Serum Vitamin D levels with Hypertension
}

\section{Maryam Rao ${ }^{1}$, Tabinda Kazmi ${ }^{2}$, Muhammad Imran ${ }^{3}$, Aamir Mahmood Farooqi ${ }^{4}$, Akif Mahmud ${ }^{5}$, Qanita Mahmud ${ }^{6}$}

\begin{abstract}
Objectives: The objective of our study was to identify the effect of low serum vitamin D levels on hypertension \& to make a comparison of levels of vitamin D in hypertensives and healthy individuals.

Materials and Method: A cross-sectional comparative study carried out in Cardiology Department of Shaikh Zayed Hospital and Punjab Institute of Cardiology, which involved measurement and comparison of serum Vitamin D levels in 64 subjects, categorized in two groups; hypertensives and normotensives, in order to establish a potential association between vitamin D levels and hypertension.

Results: Our study showed that Serum Vitamin D was equally sub-optimal in all participants of study, including both hypertensive group and the healthy controls. As a result of this finding, a clear pattern of association of vitamin D deficiency with hypertension could not be seen. Mean \pm SD Vitamin D levels was $19.5 \pm 10.0 \mathrm{ng} / \mathrm{L}$ in hypertensives and $19.5 \pm 13.6$ in healthy individuals. This study did not lead to establishment of an association between hypertension and vitamin D levels.

Conclusion: Because of deficiency of Vitamin D in general population in this region, our study failed to show an association between Vitamin D and hypertension. Further exploration is needed in this regard.

Key words: Hypertension, serum Vitamin D levels
\end{abstract}

\section{Introduction}

$\mathrm{V}$ itamins are a class of chemical substances that are present in small amounts in food. It can be classified both as a nutrient as well as a vitamin. It is naturally synthesized in the skin in the presence of ultraviolet $\mathrm{B}$ radiations of sunlight during mid-day, particularly at high altitudes. ${ }^{1,2}$

Till recent past, vitamin $\mathrm{D}$ was considered to be involved in calcium metabolism, causing increased absorption of calcium from intestine, thereby promoting bone growth and mineralization. ${ }^{3}$ Vitamin D deficiency can cause reduction of bone mass, thereby resulting in osteoporosis and osteomalacia in adults and rickets in children. ${ }^{2}$ With further advancement in

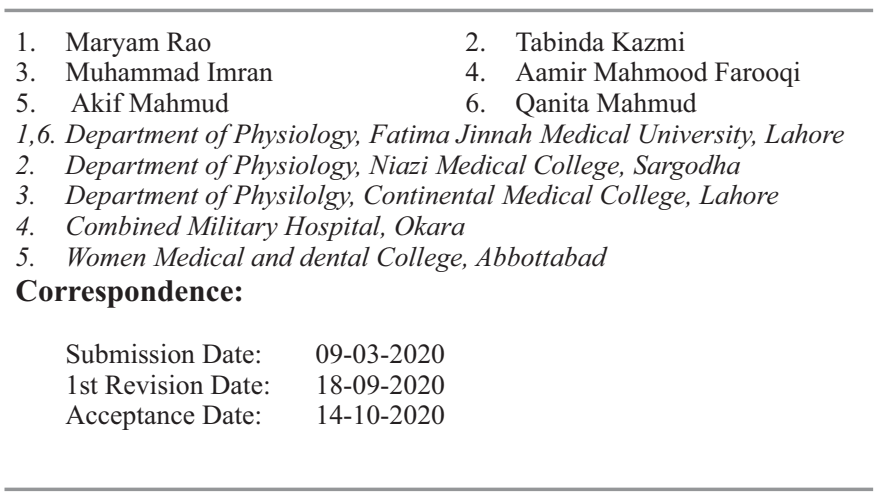

research, now vitamin $\mathrm{D}$ is considered to play remarkable role in improving muscle strength, prevention and improvement in survival of cancers, regulating immune mediated disorders like rheumatoid arthritis, multiple sclerosis, diabetes mellitus, systemic lupus erythematosus and inflammatory bowel disease. ${ }^{4}$

Besides other important metabolic effects of vitamin $\mathrm{D}$, it is considered to play a very important role in regulation of renin-angiotensin system of blood pressure regulation. This is the major blood pressure control mechanism. ${ }^{5}$ It is believed that vitamin D deficiency, somehow, causes the upregulation of renin angiotensin system, thereby, increasing the probability of development of hypertension. Similarly, vitamin D produces direct effects on vasculature, protecting its endothelium from damaging effects of advanced glycation end products and decreasing the atherosclerotic and inflammation causing factors. ${ }^{6}$ Vitamin D deficiency associated with hyperparathyroidism can also lead to arterial calcification, thereby, contributing to hypertension. ${ }^{7}$

All these effects indicate widespread presence of vitamin D receptors at sites like leucocytes, vascular 
smooth muscle cells, vascular endothelium and even juxtaglomerular cells of kidneys. ${ }^{8}$

Since vitamin D deficiency is very common in our part of the world owing to high amount of melanin in skin, which can actually decrease synthesis of vitamin $\mathrm{D}$ in response to ultraviolet $\mathrm{B}$ radiation by $99 \%$, it is very important to understand all its implication. ${ }^{9}$ This study aims to identify the possible effect of vitamin D deficiency in pathogenesis of hypertension so that its supplementation can be instituted, if such an association is established.

\section{Methods}

This cross-sectional comparative study was carried out in Cardiology Department, Shaikh Zayed Hospital \& Punjab Institute of Cardiology and Physiology Department of Shaikh Zayed Postgraduate Medical Institute, Lahore, after seeking permission from concerned authorities. The study population consisted of hypertensive patients above 40 years of age and age \& gender matched healthy controls while the patients of renal disorders or those on vitamin D supplementation were excluded from the study.

A sample population of 32 hypertensive and 32 healthy control subjects was used to compare their vitamin D levels. A convenient, non-probability sampling technique was used and blood samples of patients visiting outpatient department were taken after detailed history and examination. A detailed informed consent was taken from participants of study and later on, report of their serum vitamin D levels was mailed to them.

Estimation of vitamin D levels was done using ELISA technique after centrifuging \& separating serum.

\section{Results}

The study was conducted on 64 participants. Of these, 32 participants were hypertensive (HTN), and 32 were healthy controls without any known cardiovascular disease. Results were analyzed with the help of SPSS (version 20). Data for levels of serum vitamin $\mathrm{D}$ was expressed by using Mean $+\mathrm{SD}$ in case of both groups.

Variables among two groups were compared by using $\mathrm{t}$-test. Data pertaining to deficiency of vitamin $\mathrm{D}$ was shown in terms of percentage and frequency. Vitamin D levels were categorized for comparison with the help of chi-square test.

Mean age in case of hypertensives and controls was $52.8 \pm 6.4$ and $46.9 \pm 6.8$ years respectively. No significant difference was seen in gender distribution between the groups ( $p$-value 0.453 ).

As shown in fig.1, body mass index ranged from a minimum of 22.6 to a maximum of 34 in hypertensive group, which is quite comparable to BMI ranging from 19 to 33.4 of healthy control group. This difference was insignificant with $\mathrm{p}$-value 0.157 . These statistical findingsare tabulated in table 1.

History of hypertension in family was seen for $25(78.1 \%)$ of healthy participants and for $18(56.2 \%)$ cases of hypertension.

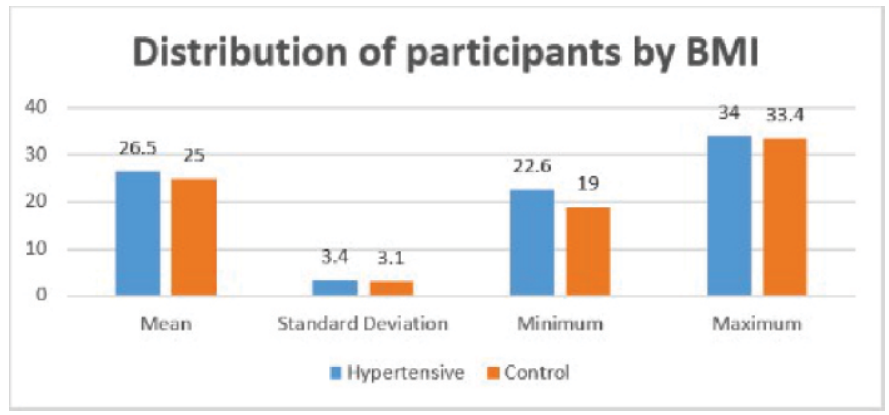

Fig.1: Distribution of Participants According to BMI

As shown in fig. $1 \&$ table 2 , mean level of vitamin D was $19.5 \pm 10.0$ in hypertensive group and $19.5 \pm 13.6$ $\mathrm{ng} / \mathrm{ml}$ in control group. Therefore, no significant difference was found among two groups with a pvalue of 0.983 . For further analysis, vitamin $\mathrm{D}$ level was divided in three categories, i.e. $<15,15-25$ and $\geq$ 25 and comparison was made using chi-square test, as shown in table 3 . There were $12(37.5 \%)$ hypertensives with deficient vitamin D levels and similarly, $13(40.6 \%)$ healthy participants showed deficient levels. In short, no difference in vitamin D status was evident between two groups with p-value 0.976.

Fig.2: Showing Comparison of Serum Vitamin D Levels in Two Groups

\section{Discussion}

This is one of the pioneer studies in our setup to explore the physiological effects of vitamin D. In this study, we aimed to compare the serum levels of 
$25(\mathrm{OH}) \mathrm{D}$ in 2 groups which were age and gender matched. One group included hypertensive patients and other one comprised of healthy control subjects.

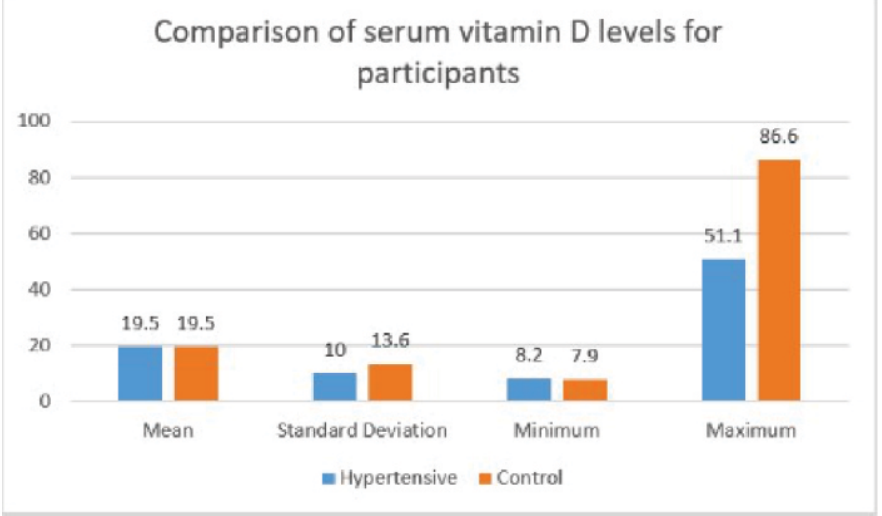

Serum vitamin D levels were found to be low in both hypertensive patients and healthy controls, thereby

Table 1: Distribution of Participants According to BMI

\begin{tabular}{lcccc}
\hline \multicolumn{5}{c}{ BMI } \\
& Mean & $\begin{array}{c}\text { Standard } \\
\text { Deviation }\end{array}$ & Minimum & Maximum \\
\hline Hypertensive & 26.5 & 3.4 & 22.6 & 34 \\
Control & 25 & 3.1 & 19 & 33.4 \\
\hline
\end{tabular}

Table 2: Showing Comparison of Serum Vitamin D level Between Two Groups

\begin{tabular}{lcccc}
\hline & Mean & $\begin{array}{c}\text { Standard } \\
\text { Deviation }\end{array}$ & Minimum & Maximum \\
\hline Hypertensive & 19.5 & 10 & 8.2 & 51.1 \\
Control & 19.5 & 13.6 & 7.9 & 86.6 \\
\hline
\end{tabular}

Table 3: Showing Comparison of Categorized Vitamin D Levels Using Chi-square Test

\begin{tabular}{lcccccccc}
\hline & \multicolumn{1}{c}{ SERUM VITAMIN D LEVELS (ng/ml) } \\
(Binned)
\end{tabular}

not suggesting any association of deficiency in cases and healthy subjects. In this study, mean vitamin D level was measured to be $19.5 \pm 10 \mathrm{ng} / \mathrm{ml}$ in hypertensive patients and $19.5 \pm 13.6 \mathrm{ng} / \mathrm{ml}$ in healthy controls.

Optimal serum vitamin D level, according to various studies is considered to be more than $30 \mathrm{ng} / \mathrm{ml}$. ${ }^{10}$ One study suggests optimal level of vitamin D of around $40 \mathrm{ng} / \mathrm{ml} .^{11}$ Few studies recommend an even higher optimal level but overall,30 $\mathrm{ng} / \mathrm{ml}$ is considered to be the lowest cut-off point. This study found the level of vitamin $\mathrm{D}$ in healthy controls equal to $19.5 \pm 13.6$ $\mathrm{ng} / \mathrm{L}$, which is certainly sub-optimal.This level is considered to be in insufficient range according to most studies. ${ }^{12,13}$ Nowadays, deficiency of vitamin Dis a common health problem worldwide which can be a consequence of insufficient vitamin $\mathrm{D}$ in diet, less exposure to sunlight, obesity, age, over use of sunscreens \& other medication usage that can impair vitamin D absorption. ${ }^{14}$ A study carried out at Washington University, revealed vitamin D levels to be insufficient/deficient in $57.5 \%$ subjects. $^{15}$ In addition to the global problem, South Asian population is even at higher risk of Vitamin D deficiency. This is due to the presence of more melanin beneath the skin that impairs synthesis process of vitamin D, insufficient sunlight exposure particularly in female gender, owing to social and cultural issues, suboptimalfood quality and lastly, selection of improper food that lacks in required vitamins reflecting poverty and ignorance.

Numerous studies conducted so far, have indicated a potential link between vitamin D deficiency \& hypertension, still none of such studies have been done in South Asian region. One of such studies was carried out in Iran, trying to establish a relationship between serum vitamin $\mathrm{D}$ levels and hypertension. This study revealed $67 \%$ hypertensive subjects having vitamin D deficiency. Further, the severity of hypertension was relatable to deficiency of vitamin D levels. ${ }^{16}$ A cohort study conducted by Form an et also revealed vitamin $\mathrm{D}$ levels to be inversely proportional to incident hypertension and this relationship was unaffected by BMI, age, race, physical exertion and menopausal status. ${ }^{6}$ One such study was carried out on American Blacks which highlighted drastic improvement in blood pressure control after supplementation of vitamin $\mathrm{D}$, for three consecutive months. ' Likewise, one more study conducted on White population of American revealed a significant inverse link of vitamin D level and category of hypertension, in the range from normotensive to stage 2 hypertension. ${ }^{17}$ Another study done recently by Wang et al revealed that vitamin D less than $15 \mathrm{ng} / \mathrm{ml}$ is related to increased hypertension $\operatorname{rate}(p=0.015)$. Detailed analysis revealed an even stronger relationship to exist between vitamin D and risk of cardiovascular events in hypertensives $(p=0.003){ }^{18}$ Multiple prospective studies have been conducted 
with measurement of initial vitamin D level in order to explore long term risk of cardiovascular complications in subjects lacking prior history of cardiovascular problems. One such study revealed a 2-fold increased rate of myocardial infarction in subjects with vitamin $\mathrm{D}$ deficiency (levels $<15 \mathrm{ng} / \mathrm{ml}$ ) during a follow up of 10 years. ${ }^{19}$ Similarly, a large Framingham Studyalso revealed an association between low vitamin D levels and serious cardiovascular disease events, with risk reaching $53 \%-80 \%{ }^{20}$ On the contrary, certain researches failed to provide any inverse relationship between vitamin D and hypertension. Infact, one study even highlighted a positive correlation to exist between vitamin D levels\&blood pressure. $^{19}$ Undoubtedly, different studies reveal variable impact of vitamin $\mathrm{D}$ on hypertensive and normotensive individuals. This shows that effect of vitamin $\mathrm{D}$ on hypertension is still an area of concern which will require a drastic exploration \& multiple comprehensive researches, before a final deduction is made. $^{16}$

This study could not establish a inverse association between risk of hypertension and serum vitamin D levels, which is attributed to sub-optimal levels of serum vitamin D even in healthy population.

\section{Conclusion}

This study resulted in following conclusions:

1) Serum vitamin $D$ is generally deficient or insufficient in our population.

2) The association between serum vitamin $D$ level and hypertension could not be established.

3) Exploration of effects of vitamin D deficiency needs further large scale studies.

\section{Limitations:}

Our study had a few limitations:

1) Sample size was not large enough to give more conclusive results.

2) To obtain more reliable results, all other possible risk factors of cardiovascular diseases must be excluded from the study.

\section{Author's Contributions}

MR: Conducted and presented the research at a conference
TK: Questionaire design, conduction

MI: Initial Draft of manuscript

AF: Reviewed and improved the initial draft of manuscript

AM: Table and graph Design,Interpreted results

QM:Planned, Conducted and supervised the research

\section{References}

1. Garriguet D. Bone health: Osteoporosis, calcium and vitamin D. Statistica Canada. 2011;22:01-8.

2. Milovanovic Z.Vitamin D deficiency and its importance-A global problem of today, realistic or not? Ser J Exp Clin Res. 2016;17(4):1-10.

3. Basit S. Vitamin D in health and disease: a literature review. British Journal of Biomedical Science. 2013; 70(4):161-72.

4. Spedding S. Vitamin D and Human Health: Celebrating Diversity. Nutrients. 2014;6:11-4.

5. Iftikhar Ullah M, Uwaifo G, Nicholas W and Koch C. Does Vitamin D Deficiency Cause Hypertension? Current Evidence from Clinical Studies and Potential Mechanisms. International Journal of Endocrinology. 2010;2010:01-11.

6. Zanchetti A. Predictive, mechanistic and therapeutic studies on hypertension and cardiovascular morbidity and mortality. Journal of Hypertension. 2014; 32: $1549-50$.

7. Gupta M,Patil R,Khan I and Kumar S. The Prevalence of Obesity and Hypertension in Urban Tamilnadu. Journal of Clinical and Diagnostic Research. 2011; 5:586-8.

8. Kienreich K, Grubber M, Tomaschitz A, Schmid J,Verheyen N,Rutters F,Dekker J and Pilz S.Vitamin $\mathrm{D}$, arterial hypertension and cerebrovascular disease. Indian J Med Res.2013;137:669-79.

9. Forman J,Scott J,Kimmie Ng,Drake B,Suarez E,Hayden D,Bennett G,Chandler P,Hollis B,Emmons K,Giovannucci E,Fuchs $\mathrm{C}$ and Chan A.Effect of Vitamin D Supplementation on Blood Pressure in Blacks. Hypertension. 2013;61:779-85.

10. Joshi D, Center J and Eisman J. Vitamin D deficiency in adults. Australian Prescriber. 2010;33:103-6.

11. Menyar A, Rahil A, Dousa K, Ibrahim W, Ibrahim T, Khalifa R and Abur-Rehman M. Low Vitamin D and Cardiovascular Risk Factors in Mals and Females from a Sunny, Rich Country. The Open Cardiovascular Journal. 2012;6:76-80.

12. Thacter T and Clarke B. Vitamin D Insufficiency. Mayo Clin Proc. 2011;86:50-60.

13. Chaudhuri J, Mridula R, Anamika A, Boddu D, Misra $\mathrm{P}$, Lingaiah A, Balaraju B and Bandaru S. Deficiency 
of 25-Hydroxyvitamin D and Dyslipidemia in Indian Subjects. Journal of Lipids. 2013;13:623420.

14. Kienreich K, Tomaschitz A, Verheyen N, Pieber T, Gaksch M, Grubler M and Pilz S. Vitamin D and Cardiovascular Disease. Nutrients. 2013;5:3005-21.

15. Larijani F, Mohatmedi S, Keshtkhar A, Khashayar P, Koleini Z, Rahim F and Larijani B. The Relation between Serum Vitamin D levels and Blood Pressure: A Population Based Study. Acta Medica Iranica. 2014;52:290-7.

16. Judd S,Nanes M,Ziegler T,Wilson P and Tangpricha V.Optimal vitamin D status attenuates the age-associated increase in systolic blood pressure in white Americans: results from the third National Health and Nutrition Examination Survey. Am J Clin Nutr.
2008;87:136-41.

17. Judd S and Tangpricha V. Vitamin D Deficiency and Risk for Cardiovascular Disease. Am J Med Sci. 2009;338;40-4.

18. Lee J, O'Keefe J, Bell D, Hensrud D and Holick M. Vitamin D Deficiency-An Important, Commonand Easily Treatable Cardiovascular Risk Factor.J Am Coll Cardiol. 2008;52:1949-56.

19. Scragg R,Sowers $M$ and Bell C.Serum 25hydroxyvitamin $\mathrm{D}$, ethnicity and blood pressure in the third national health and nutrition examination survey. Am J Hypertens. 2007;20:713-9.

20. Available at http://www.sciencedaily.com/ releases/ 2013/06/130610192638.htm. Accessed on September 18,2015. 